\title{
La calidad universitaria que debemos tener en el bicentenario
}

\section{The university quality that we must have in the bicentenary}

DOI: $10.46932 / \mathrm{sfjdv} 2 \mathrm{n} 4-073$

Received in: May 1st, 2021

Accepted in: Jun 30th, 2021

\author{
Pedro Julián Ormeño Carmona \\ Doctor \\ Institución actual: Universidad Nacional de Ucayali
}

Dirección completa: Car. Federico Basadre KM. REF: Ucayali Coronel Portillo Calleria

Correo electrónico: pedro_ormeno@unu.edu.pe

Judith Davila Talepcio

Doctor

Institución actual: I.E. 65058 Los Libertadores de América

Dirección completa: JR. Pachacutec MZ.31 LT.1 REF: Ucayali Coronel Portillo Calleria

Correo electrónico: jdavilat79@gmail.com

\section{Manuel Rocha Gonzales}

Doctor

Institución actual: Universidad Nacional de Ucayali

Dirección completa: Car. Federico Basadre KM. REF: Ucayali Coronel Portillo Calleria

Correo electrónico: manuel_rocha@unu.edu.pe

\section{José Ángel Meneses Jiménez \\ Doctor}

Institución actual: Universidad Nacional de Ucayali

Dirección completa: Car. Federico Basadre KM. REF: Ucayali Coronel Portillo Calleria

Correo electrónico: jose_meneses@unu.edu.pe

Fernando Pasquel Flores

Magíster

Institución actual: I.E Manuel Enrique Rojas Vela

Dirección completa: Calle Ricardo Valles S/N Loreto/Ucayali/Contamana

Correo electrónico: fernandoamorpf@ hotmail.com

\section{RESUMEN}

Cercano al cumplimiento del bicentenario, es necesario reflexionar sobre esta frase: "La educación que queremos para el Perú" que subtitula el Proyecto Educativo Nacional al 2021, y que se relaciona con el título de este ensayo, tiene un relación directa con el quinto objetivo estratégico que indica que "la educación superior de calidad se convierte en un factor favorable para el desarrollo y la competitividad nacional (CNE, 2007)

Y es que la calidad de la educación superior constituye una herramienta estratégica muy valiosa para el desarrollo, por ello el gobierno debe implementar políticas públicas para hacer cambios trascendentales con mayor inversión, fortalecer las capacidades de gerentes y docentes, e implementar un sistema de gestión de la calidad, promover una cultura de mejora continua. 
La calidad que se necesita para todos los sectores es una demanda nacional que no se puede postergar más, y estando cerca de un proceso electoral, es válido exigirlo a los candidatos de hoy, que si son electos implementen políticas públicas de promoción, promoción y desarrollo de una cultura de calidad a favor de nuestro país, para esta y las próximas generaciones.

Palabras clave: acreditación, bicentenario, educación, mejora continua.

\begin{abstract}
Nearing the bicentennial, it is necessary to reflect on this phrase: "The education we want for Peru" that subtitles the National Education Project to 2021, and which is related to the title of this essay, has a direct relationship with the fifth strategic objective that indicates that "quality higher education becomes a favorable factor for development and national competitiveness (CNE, 2007).

The quality of higher education is a very valuable strategic tool for development, which is why the government must implement public policies to make transcendental changes with greater investment, strengthen the capacities of managers and teachers, implement a quality management system, and promote a culture of continuous improvement.

The quality that is needed for all sectors is a national demand that cannot be postponed any longer, and being close to an electoral process, it is valid to demand that today's candidates, if elected, implement public policies to promote, foster and develop a culture of quality in favor of our country, for this and the next generations.
\end{abstract}

Key words: accreditation, bicentennial, education, continuous improvement.

\title{
1 INTRODUCCIÓN
}

Estando próximos al cumplimiento del bicentenario del país, es necesario reflexionar sobre esta frase: "La educación que queremos para el Perú" que subtitula el Proyecto Educativo Nacional al 2021, donde señala que se aspira a lograr la calidad en todos los niveles educativos, en el quinto objetivo estratégico señala que:

La educación superior de calidad se convierte en factor favorable para el desarrollo y la competitividad nacional, por ello es necesario asegurar la calidad de la educación superior y su aporte al desarrollo socioeconómico y cultural en base a prioridades, así como la inserción competitiva en la economía mundial (Consejo Nacional de Educación 2007, p.47)

En ese sentido el presente ensayo del eje temático: Universidad y sociedad, pretende describir la importancia de la calidad de las universidades públicas y privadas, por ser un factor determinante para el crecimiento económico en nuestro país. La calidad de la educación superior, se constituye en una herramienta estratégica muy valiosa para el desarrollo integral de cualquier país, prueba de ello son los países que se encuentran muy bien ubicados en el ranking sobre calidad de vida, desarrollo urbano, desarrollo humano y etc.

Esta calidad se debe a que sus gobiernos de turno decidieron hacer cambios trascendentales en las políticas educativas con mayor inversión, para el fortalecimiento de capacidades a directivos y docentes; 
y sobre todo porque implementaron un sistema de gestión de calidad, para establecer una línea base situacional, y tomar pertinentes decisiones administrativas para promover una cultura de calidad en favor del desarrollo educativo y por ende del país.

\section{DESARROLLO}

Según la Superintendencia nacional de Educación Superior Universitaria [SUNEDU] (2015) establece que el proceso de licenciamiento institucional tiene como bases la Ley Universitaria №30220, vigente desde julio de 2014, y el modelo de licenciamiento elaborado por la SUNEDU, que fue aprobado y difundido en noviembre de 2015. En donde se establece las condiciones básicas de calidad (CBC) que todas las universidades públicas y privadas deben cumplir para continuar con su oferta de servicios educativos, Así mismo dichas normas establecen el procedimiento que se debe seguir ante la SUNEDU para obtener la licencia institucional que las habilita para brindar el servicio formativo de nivel superior en pre y posgrado. Por ello las universidades peruanas tienen la responsabilidad funcional de cumplir con estas condiciones básicas de calidad en el proceso de enseñanza-aprendizaje en favor de sus estudiantes que son la razón de ser.

En ese sentido el Ministerio de Educación (2015) en el marco de la Ley Universitaria 30220 aprobó el DS-016-2015-MINEDU, Política de Aseguramiento de la calidad de la Educación Superior Universitaria, con el objetivo de garantizar un servicio educativo universitario de calidad, que ofrezca una formación integral y de perfeccionamiento continuo, centrado en el logro de un desempeño profesional competente $\mathrm{y}$, en la incorporación de valores ciudadanos que permitan una reflexión académica del país, a través de la investigación; y con el objetivo técnico de organización sistémica de todos los actores involucrados en la educación superior universitaria y el desarrollo de un Sistema de Aseguramiento de la Calidad (SAC).

La Política establece como pilares para la construcción de un Sistema de Aseguramiento de la Calidad: la Acreditación para la mejora continua, entendida como la garantía socialmente reconocida que brinda el estado sobre la calidad de la institución o un programa de estudios conducente a obtener un grado académico; y el Licenciamiento como garantía de condiciones básicas de calidad, entendido como la verificación y control de dichas condiciones que permite autorizar la provisión del servicio educativo superior universitario.

En la actualidad en nuestro país 46 universidades públicas y 50 universidades privadas han demostrado cumplir con los indicadores de calidad, obteniendo su licenciamiento institucional. así mismo 03 universidades nacionales y 44 universidades privadas, no obtuvieron el licenciamiento debido al incumplimiento de las condiciones básicas de calidad, debiendo de cesar sus funciones en un plazo de dos 
años, sin embargo, debido a la pandemia del COVID 19, la Sunedu amplio su funcionamiento a 5 años. El licenciamiento ha instaurado un proceso de orden, marcó "un antes y un después" en todas las universidades del país, ha sido una forma de mirarse, de identificar sus fortalezas, debilidades y desafíos para su crecimiento, fue una oportunidad para ponerse en orden y asegurar la calidad y la mejora continua, que les permitió ganar reconocimiento y destacar su oferta.

El Sistema Nacional de Evaluación Acreditación y Certificación de la Calidad Superior [SINEACE], (20216) aprobó con Resolución de Presidencia del Consejo Directivo Ad Doc N 175-2016SINEACE/CDAH-P, de fecha 24 de noviembre 2016, la versión final del Modelo de Acreditación para Programas de Estudio de Educación Superior Universitaria, este nuevo modelo concibe la evaluación de la calidad como un proceso formativo que ofrece a las instituciones oportunidades para analizar su quehacer, introducir cambios para mejorar de manera progresiva, permanente y sostenida, fortalecer su capacidad de autorregulación e instalar una cultura de calidad institucional a través de la mejora continua.

En este contexto el SINEACE (20218, p. 10) ha identificado una estructura para el modelo, que incluye cuatro categorías generales o dimensiones. La primera dimensión de gestión estratégica referente al planeamiento y la dirección de un programa académico que asegure el proceso de mejora continua, alineados a la misión y visión y los propósitos institucionales de la universidad, construidos con la participación de toda la comunidad universitaria, en especial de los grupos de interés para asegurar que la oferta académica sea pertinente a la demanda social, con planes de desarrollo regional, nacional e internacional sostenibles en el tiempo. (SINEACE, 2018, p. 13-41)

La segunda dimensión central de formación integral, referido al proceso de enseñanza aprendizaje con énfasis en el plan de estudios que tiene que ser evaluado y actualizado de forma oportuna, para definir las competencias que debe tener el estudiantes cuando ingresa y egresa, siendo el perfil de egreso la columna vertebral del plan de estudio, así mismo esta dimensión considera la articulación del plan de estudio con la investigación, docentes y responsabilidad social para asegurar la formación integral y desarrollo de competencias de los estudiantes.

Es importante que las universidades cuenten con docentes, que tengan los conocimientos, experiencia académica y profesional, así como una conducta ética para asegurar el logro de las competencias del perfil de egreso y objetivos educacionales, así mismo es importante que los docentes realicen investigaciones en el área disciplinar o programa al que pertenecen de acuerdo a las líneas de investigación de cada carrera profesional, sim embargo esto se convierte en una limitación puesto que un mínimo porcentaje de docentes en las universidades realizan investigaciones. 
Y esta dificultad también se traspasa a los estudiantes quienes muy pocos son los que realizan sus propias investigaciones para obtener su titulo profesional, la mayoría de veces son terceros quienes realizan estas investigaciones. La investigación realizada en una universidad puede dar señales sobre la cantidad y calidad de los conocimientos científicos que poseen los docentes y, por tanto, del valor de los cursos que dictan. (SINEACE, 2018, p.39-108)

La tercera dimensión de soporte institucional con referencia al equipamiento, recursos e infraestructura centrados en el bienestar de los integrantes de la universidad. Los programas de bienestar deben contribuir a la formación integral y favorecer a la articulación armoniosa de los proyectos personales de vida en el ámbito del estudio y el trabajo, las universidades deben garantizar una adecuada infraestructura con salones de clase, oficinas, laboratorios, talleres y equipamiento pertinentes para su desarrollo y formación integral de los estudiantes; además, necesita contar con un sistema que pueda mantener informada a la comunidad universitaria y a la sociedad. (SINEACE, 2018, p.109-125)

La cuarta dimensión de resultados; referentes a la verificación los objetivos educacionales que hace referencia a los logros profesionales luego de un determinado tiempo de haber egresado; y el perfil de egreso, que considera todas las competencias, cualidades, habilidades y valores que deben lograr los estudiantes al finalizar el proceso de formación. (SINEACE, 2018, p.129-139)

En la lógica del modelo de acreditación, el perfil de egreso se convierte en un eje central y articulador del programa de estudios. El perfil de egreso se identifica como parte de la gestión estratégica y conduce además la planificación del programa; orienta el proceso de formación integral; y el logro del mismo debe verificarse en cada egresado. La matriz de evaluación está organizada en 4 dimensiones, 12 factores y 34 estándares que se acompañan de criterios a evaluar. (SINEACE, 2018)

Si bien es cierto que la Ley del Sistema Nacional de Evaluación, Acreditación y Certificación de la Calidad Educativa (SINEACE) y su reglamento establecen los procesos de evaluación de la calidad educativa con fines de acreditación en nuestro país; este escenario compromete a toda la comunidad universitaria, autoridades, docentes, alumnos, administrativos, egresados y a grupos de interés a tomar una actitud responsable para el fortalecimiento de los procesos de formación profesional acorde a los estándares establecidos en el modelo de acreditación de programas de estudios de educación superior universitaria del SINEACE.

El actual contexto de la Covid-19 ha puesto en evidencia la fragilidad estructural de los sectores de salud y educación, en donde existen brechas de tecnología, conectividad y poco desarrollo de competencias profesionales para entornos virtuales; a pesar que las universidades estaban licenciadas y garantizaron el cumplimiento de algunos estándares de calidad, no estaban preparadas para brindar una enseñanza a distancia. 
Esto debido a la falta de implementación de plataformas virtuales que pudieran ser usadas por todos los estudiantes, el desconocimiento de los docentes para usar e implementar estas plataformas para realizar sus clases virtuales, estudiantes sin conectividad en sus hogares y sin un dispositivo móvil o una laptop para acceder a las clases online, que ha conllevado que muchos estudiantes no puedan continuar con sus estudios, si bien es cierto muchas universidades ofrecieron a los estudiantes con bajos recursos económicos la posibilidad de prestarles o donarles ordenadores portátiles, no pudieron cobertura a todos los estudiantes, esto genera la incertidumbre si las universidades están brindando una educación de calidad a los futuros profesionales del país.

En ese escenario el mercado laboral espera que las instituciones de nivel superior como las universidades, le brinden un producto (egresado) acorde a sus necesidades que tienen, con un alto nivel de competencias y que cumpla con sus expectativas, para el logro de sus objetivos organizacionales. Esta perspectiva es acorde con Garbanzo (2012, p. 218) quien señala que la sociedad actual espera de las instituciones de educación una capacidad de respuesta en forma competitiva a la sociedad del conocimiento, asegurando así su desarrollo y sostenibilidad social, lo cual requiere un proyecto educativo y social más inclusivo.

Por ello en este tiempo es importante diseñar e implementar un sistema de gestión de la calidad acorde al actual contexto universitario, donde se desarrollen procesos de planificación, control y mejoramiento continuo, de manera que se obtenga la satisfacción de los usuarios. Según Guerra y Jaya (2016, p.36) conceptúan la calidad considerando la norma internacional ISO 9000:2015 como: “el grado en que un conjunto de características inherentes al servicio educativo cumple con los requisitos asociados a las necesidades y expectativas de los grupos de interés".

Así mismo Guerra y Jaya (2016, p.36) menciona que:

La norma ISO 9001: 2015 favorece enfoque estratégico fortaleciendo el pensamiento basado en el riesgo para planificar y ejecutar acciones dentro de un sistema de gestión calidad que satisfaga de manera equilibrada los requerimientos en las tres dimensiones de la educación superior: docencia, investigación y extensión.

Por ello es importante señalar que la norma ISO 9001, tiene una estructura práctica para el diseño e implementación de sistemas de gestión de la calidad en cualquier contexto organizacional (Fontalvo, 2000).

En ese sentido es válido decir que se debería implementar un Sistema de Gestión de la Calidad ISO 9001:2015 en la academia, y es que las condiciones básicas de calidad exigidas por SUNEDU, no es suficiente, los programas académicos deben ser acreditados por una entidad acreditadora, siendo el factor clave para conseguirlo el compromiso institucional de sus autoridades en la implementación de un Sistema de Gestión de la Calidad que debería ser considerado como una política de gestión institucional establecida por el MINEDU, que debe tener en cuenta que la implementación de una cultura de calidad, debe ser una 
condición para toda la gestión administrativa, formativo y de responsabilidad social, que debe hacer la academia en su desarrollo institucional, para poder atender las demandas del sector empresarial, social y cultural, las cuales deben estar en un sistema de información de libre acceso para que se puedan hacer investigaciones aplicadas y reducir o resolver los problemas que se irán presentando. La calidad que se necesita para todos los sectores, es una demanda nacional que no se puede postergar más, y estando próximo a un proceso electoral de segunda vuelta, es válido exigírselo mediante un compromiso público a los hoy candidatos, que de ser elegidos electos se implementen políticas públicas de promoción y desarrollo de una cultura de calidad en la academia para que este bicentenario sea el inicio de un nuevo tiempo de cambios en nuestro país.

\section{CONCLUSIONES}

Podemos concluir que la calidad de la universidad se evidencia cuando:

La Universidad se convierte en una comunidad formativa de aprendizaje profesional integrado por docentes y estudiantes que trabajan en colaboración y dialogo reflexivo para el logro de las competencias profesionales. Realizando permanentemente investigaciones e innovaciones, que fomenten en dominio del conocimiento científico y tecnológico que conlleve a la reflexión en profundidad

La Universidad debe formar líderes capaces de integrar a los diferentes grupos humanos, con conocimiento de su realidad regional, nacional e internacional para promover el desarrollo económico del país.

Las universidades deben implementar un Sistema de Gestión de Calidad ISO 9001:2015, como exigencia institucional por parte del MINEDU, para garantizar el cumplimiento del perfil profesional de los estudiantes, el de desarrollar proyectos de responsabilidad social acordes a las necesidades de sus zonas de influencias y para garantizar el desarrollo de las líneas de investigación que contribuyan a la solución de las problemas sociales. 


\section{REFERENCIAS BIBLIOGRAFICAS}

Consejo Nacional de Educación, (2007), Proyecto educativo Nacional al 2021, Lima.

Fontalvo, H. (2000), La gestión avanzada de la calidad: metodologías eficaces para el diseño, implementación y mejoramiento de un sistema de gestión de la calidad, Cartagena: Corporación para la gestión del conocimiento ASD 2000.

Garbanzo, G. (2012), Educación superior pública en América Latina: características y desafíos. Revista GUAL, 5(1), 216-227

Guerra, R. Jaya, A. (2016), El papel de los Stakeholders en la gestión de la calidad universitaria: el enfoque de la ISO 9001:2015, Revista Caribeña de Ciencias Sociales, 5(12), 1-15 .

Superintendencia nacional de Educación Superior Universitaria [SUNEDU] (2015), El Modelo de Licenciamiento y su implementación. https://www.sunedu.gob.pe/modelo-licenciamiento-institucional/.

Ministerio de Educación (2015), Aprobación de la Política de Aseguramiento de la Calidad de la Educación Superior Universitaria. Decreto supremo Nº16-2015-MINEDU, Lima.

Sistema Nacional de Evaluación Acreditación y Certificación de la Calidad Superior (2016), Modelo de Acreditación para Programas de Estudio de Educación Superior Universitaria. Lima.

Sistema Nacional de Evaluación Acreditación y Certificación de la Calidad Superior (2018), Explicación de estándares del modelo de acreditación de programas de estudios de educación superior universitaria. Lima: Tarea Asociación Gráfica Educativa 